\section{Genomic profiling of primary histiocytic sarcoma reveals two molecular subgroups}

\author{
Caoimhe Egan, ${ }^{1}$ Alina Nicolae,${ }^{1}$ Justin Lack, ${ }^{2}$ Hye-Jung Chung, ${ }^{1}$ \\ Shannon Skarshaug, ${ }^{1}$ Thu Anh Pham, ${ }^{1}$ Winnifred Navarro, ${ }^{1}$ Zied Abdullaev, ${ }^{1}$ \\ Nadine S. Aguilera, ${ }^{3}$ Liqiang $\mathrm{Xi},{ }^{1}$ Svetlana Pack,${ }^{1}$ Stefania Pittaluga, ${ }^{1}$ \\ Elaine S. Jaffe ${ }^{1}$ and Mark Raffeld ${ }^{1}$
}

${ }^{1}$ Laboratory of Pathology, National Cancer Institute, National Institutes of Health, Bethesda, MD; ${ }^{2}$ NIAID Collaborative Bioinformatics Resource (NCBR), National Institute of Allergy and Infectious Diseases, National Institutes of Health, Bethesda, MD and ${ }^{3}$ University of Virginia Health System, Charlottesville, VA, USA
$\mathrm{H}$ istiocytic sarcoma is a rare malignant neoplasm that may occur de novo or in the context of a previous hematologic malignancy or mediastinal germ cell tumor. Here, we performed whole exome sequencing and RNA-sequencing (RNA-Seq) on 21 archival cases of primary histiocytic sarcoma. We identified a high number of genetic alterations within the RAS/RAF/MAPK pathway in 21 of 21 cases, with alterations in NF1 (6 of 21), MAP2K1 (5 of 21), PTPN11 (4 of 21), BRAF (4 of 21), KRAS (4 of 21), NRAS (1 of 21), and LZTR1 (1 of 21), including single cases with homozygous deletion of NF1, high-level amplification of PTPN11, and a novel TTYH3-BRAF fusion. Concurrent NF1 and PTPN11 mutations were present in 3 of 21 cases, and 5 of 7 cases with alterations in NF1 and/or PTPN11 had disease involving the gastrointestinal tract. Following unsupervised clustering of gene expression data, cases with NF1 and/or PTPN11 abnormalities formed a distinct tumor subgroup. A subset of NF1/PTPN11 wild-type cases had frequent mutations in B-cell lymphoma associated genes and/or clonal IG gene rearrangements. Our findings expand the current understanding of the molecular pathogenesis of this rare tumor and suggest the existence of a distinct subtype of primary histiocytic sarcoma characterized by NF1/PTPN11 alterations with predilection for the gastrointestinal tract.

\section{Introduction}

Histiocytic sarcoma (HS) is a rare and aggressive malignant neoplasm that has morphological and immunophenotypic features of mature tissue histiocytes. ${ }^{1}$ It predominantly occurs in adulthood, although any age may be affected. Sites of involvement may be nodal or extranodal, and include the gastrointestinal (GI) tract, skin, and liver. ${ }^{2}$ Histologically, the tumor cells are usually pleomorphic with cytologic atypia and can be multinucleated or have a spindled or xanthomatous morphology. The diagnosis of HS requires the demonstration of histiocytic markers (CD68, CD163, CD4 or lysozyme) and the exclusion of tumors of other lineages by negativity of immunohistochemical stains for Langerhans cells (CD1a, langerin), follicular dendritic cells (CD21, CD23, clusterin), B and T cells, cells of myeloid or epithelial lineage (MPO, CK), and melanocytic markers., ${ }^{3,4}$

Histiocytic sarcoma may arise as a primary neoplasm (pHS), but is also well described in the context of an existing or concurrently diagnosed hematologic malignancy, most frequently a follicular lymphoma, but also chronic lymphocytic leukemia (CLL) and B- or T-lymphoblastic leukemia (B-ALL/T-ALL) ${ }^{5.9}$ Rare cases have also been associated with mediastinal germ cell tumor. ${ }^{3}$ Cases arising in the context of a lymphoid neoplasm are often referred to as "secondary" HS (sHS), and frequently possess identical clonal antigen receptor gene rearrangements or occasionally identical structural events (e.g. identical IGH/BCL2 rearrangements) as in the associated lymphoma. However, on histological and immunophenotypic
Ferrata Storti Foundation
Haematologica 2020

Volume 105(4):951-960

\section{Correspondence:}

MARK RAFFELD

mraff@mail.nih.gov

Received: June 25, 2019.

Accepted: August 21, 2019.

Pre-published: August 22, 2019.

doi:10.3324/haematol.2019.230375

Check the online version for the most updated information on this article, online supplements, and information on authorship \& disclosures: www.haematologica.org/content/105/4/951

\section{(C)2020 Ferrata Storti Foundation}

Material published in Haematologica is covered by copyright. All rights are reserved to the Ferrata Storti Foundation. Use of published material is allowed under the following terms and conditions:

https://creativecommons.org/licenses/by-nc/4.0/legalcode. Copies of published material are allowed for personal or internal use. Sharing published material for non-commercial purposes is subject to the following conditions:

https://creativecommons. org/licenses/by-nc/4.0/legalcode, sect. 3. Reproducing and sharing published material for commercial purposes is not allowed without permission in writing from the publisher. 
examination they have no other evidence of lymphoid origin. ${ }^{5,6,10}$ Rather, these cases express markers of histiocytic/monocytic differentiation, but are nonetheless thought to be related to the associated B-cell neoplasm through a poorly understood process sometimes referred to as transdifferentiation $^{5}$ or origin from a common neoplastic progenitor. ${ }^{11}$ Interestingly, the presence of clonal IG gene rearrangements or a $B C L 2$ translocation is not restricted to secondary cases associated with a B-cell malignancy, as both abnormalities have also been observed in sporadic or "primary" cases of HS. ${ }^{12,13}$

In contrast to the more comprehensive studies performed in other histiocytic tumors, especially Langerhans cell histiocytosis and Erdheim-Chester disease, ${ }^{14-17}$ until recently, molecular analysis of HS has remained relatively underexplored. ${ }^{18}$ BRAF p.V600E mutations have been reported in approximately $12 \%$ of 108 published cases with molecular or immunohistochemical data, and additional alterations in members of the RAS/MAPK and PIBK/AKT pathways, including other BRAF variants, KRAS, HRAS, NRAS, MAP2K1, PIK3CA, PTPN11 and PTEN are also described (see Online Supplementary Tables $S 1$ and S2 for a complete list of references). The distinction between pHS and sHS is often not clearly defined in these studies. To better understand the genetic landscape of alterations in a well-characterized series of $\mathrm{pHS}$, we performed an integrated genomic analysis of 21 cases utilizing whole exome sequencing, whole transcriptome sequencing, and copy number analysis. Cases of sHS were intentionally excluded from this study.

\section{Methods}

\section{Case selection, IGH/BCL2 and clonality studies}

Twenty-one cases of $\mathrm{pHS}$ were identified from the files of the Hematopathology Section of the National Cancer Institute under an Institutional Review Board approved protocol (Online Supplementary Methods). The histological and immunophenotypic features and clonality characteristics of the cases are detailed in Figure 1 and Online Supplementary Table S3. DNA and RNA were isolated from formalin-fixed paraffin embedded (FFPE) tissue. Immunoglobulin (IGH and IGK) and T-cell receptor (TRG) gene rearrangement studies were performed in 19 of 21 cases and IGH/BCL2 (MBR) translocation analysis in 17 of 21 cases (Online Supplementary Methods).

\section{Whole exome sequencing}

Samples were sequenced in two groups: an initial cohort of 15 tumor samples with two matched normal samples on an Illumina HiSeq2500 with TruSeq V4 chemistry and a subsequent cohort of six tumor samples with one matched normal sample on an Illumina HiSeq3000 with TruSeq V2 chemistry (Illumina, San Diego, CA, USA). Alignment and variant calling were performed following the Center for Cancer Research Collaborative Bioinformatics Resource (CCBR) pipeline (https://github.com/CCBR/Pipeliner) as described in the Online Supplementary Methods.

\section{Variant analysis}

Germline variants were excluded in three cases with available matched normal samples. Exonic variants with a depth of coverage $\geq 20$ and a read count $\geq 6$ were retained. As matched germline samples were unavailable for most cases, we generated a targeted gene list to reduce the number of variants for review. Genes were compiled from the COSMIC Cancer Gene Census (http://cancer.sanger.ac.uk) $)^{19}$ and literature review to select disease relevant genes with a potential oncogenic role. The list was supplemented with additional genes identified by filtering the exome sequencing data to include recurrently mutated genes $(\geq 3$ samples) after removing variants based on CADD phred-like scores ${ }^{20}$ and population allele frequencies (Online Supplementary Methods). All variants involving genes in the targeted gene list were evaluated and categorized as significant based on set criteria (Online Supplementary Methods and Online Supplementary Figure S1). Variants not meeting the set criteria were excluded. Mutations were reviewed in the Integrative Genomics Viewer (IGV) ${ }^{21}$

\section{RNA sequencing}

Details of RNA library preparation, sequencing and fusion detection are described in the Online Supplementary Methods. RNASeq analysis was conducted using the CCBR RNA-Seq pipeline (https://github.com/CCBR/Pipeliner). Gene set enrichment analysis was performed using Ensemble of Gene Set Enrichment Analyses (EGSEA data version: 1.6 .0$)^{22}$ and sorted by average rank.

\section{Copy number analysis}

Nine samples were successfully assessed using the OncoScan CNV FFPE Assay (Affymetrix, Santa Clara, CA, USA) according to the manufacturer's protocol. Copy number was estimated from the exome sequencing data in the remaining cases using default settings for CNVkit v0.8.5. $5^{23}$ and PureCN v1.8.1. ${ }^{24}$ Calls from CNVkit were exported in nexus.ogt format for review and annotation in Nexus 9.0 Software (BioDiscovery, Hawthorne, CA, USA). Alterations called by both algorithms were further analyzed as described in the Online Supplementary Methods.

\section{Data sharing}

All genomic data from this study will be deposited in the dbGaP database (www.ncbinlm.nih.gov/gap) with the accession number phs001748.v1.p1.

\section{Results}

\section{Primary histiocytic sarcoma is characterized by} frequent alterations involving the RAS/MAPK pathway

Whole exome sequencing was performed on 21 cases of pHS as defined in the Online Supplementary Methods, and on three matched normal controls (His01, His08, His16), in two groups. The median coverage in the first 15 cases ranged from 106-165x, and in the second six cases from 205-305x. Sequencing depth for the three matched controls ranged from $72-143 x$. Variants were filtered as described in the Online Supplementary Methods using stringent criteria, and all candidates were individually reviewed in IGV.

Multiple and occasionally concurrent mutations involving genes of the RAS/MAPK pathway (Figure 2, Online Supplementary Table S4 and Online Supplementary Figure S2) were identified in 19 of the 21 cases. The most frequently mutated RAS/MAPK pathway genes were NF1 and MAP2K1 (5 cases each). Interestingly, 4 of 5 cases with NF1 mutations involved the GI tract, although one biopsy sequenced was a supraclavicular lymph node. Three of the cases had a single NF1 mutation (p.Q1822* [His01]; p.V1182D [His02]; p.Q1086* [His16]), whereas the other two had two mutations each (p.R304* and p.Q1775* [His12]; p.L298* and p.K660fs [His17]). Six of the seven variants were nonsense mutations or frameshift deletions 
and therefore predicted to be inactivating. The single missense mutation (p.V1182D) was predicted to be deleterious or probably damaging by functional impact algorithms SIFT ${ }^{25}$ and PolyPhen-2. ${ }^{26}$ In addition to NF1 mutations, 3 of the 5 cases showed concurrent mutations in PTPN11 (p.F71V [His01]; p.E76G [His02]; p.A72V [His12]). PTPN11 mutations were present within the autoinhibitory N-SH2 domain at amino acid residues known to be associated with a gain-of-function consequence and described in Noonan syndrome and juvenile myelomonocytic leukemia
(JMML). ${ }^{27,28}$ One case without a PTPN11 mutation [His17] had an additional mutation in GNAI2 at p.R179H, a codon previously shown to be targeted by activating mutations ${ }^{29}$ and 1 of the 5 NF1 mutated cases also had a mutation in JAK2 at p.V617F [His12]. A fourth PTPN11 mutated case at p.E76K [His18] did not have another RAS pathway mutation; however, it had high level amplification of the mutated PTPN11 allele (see below).

Additional mutations involving the RAS/MAPK pathway were detected in another 13 cases, none of which had
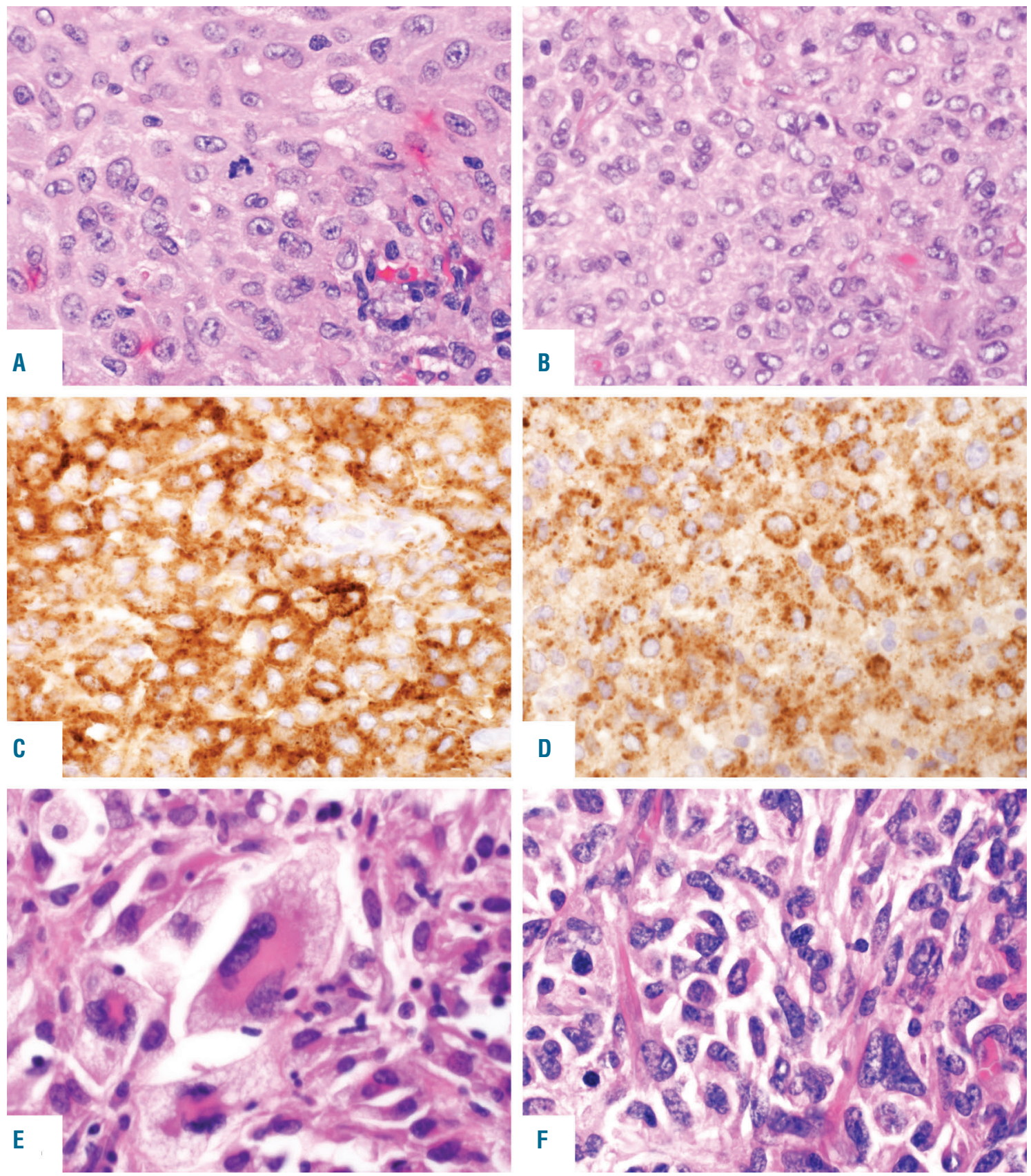

Figure 1. Histological features of primary histiocytic sarcoma (pHS). Cases involving lymph node (A) and tonsil (B) showing moderate nuclear atypia and abundant eosinophilic cytoplasm; Hematoxylin \& Eosin (H\&E) staining, original magnification x400. The cells express (C) CD163; original magnification x400 and (D) CD68; original magnification $\mathrm{x} 400$. ( $\mathrm{E}$ and $\mathrm{F}$ ) Two extranodal (gastrointestinal tract) cases showing marked nuclear pleomorphism, multinucleated cells and foam cells; H\&E stain, original magnification $\times 400$. 


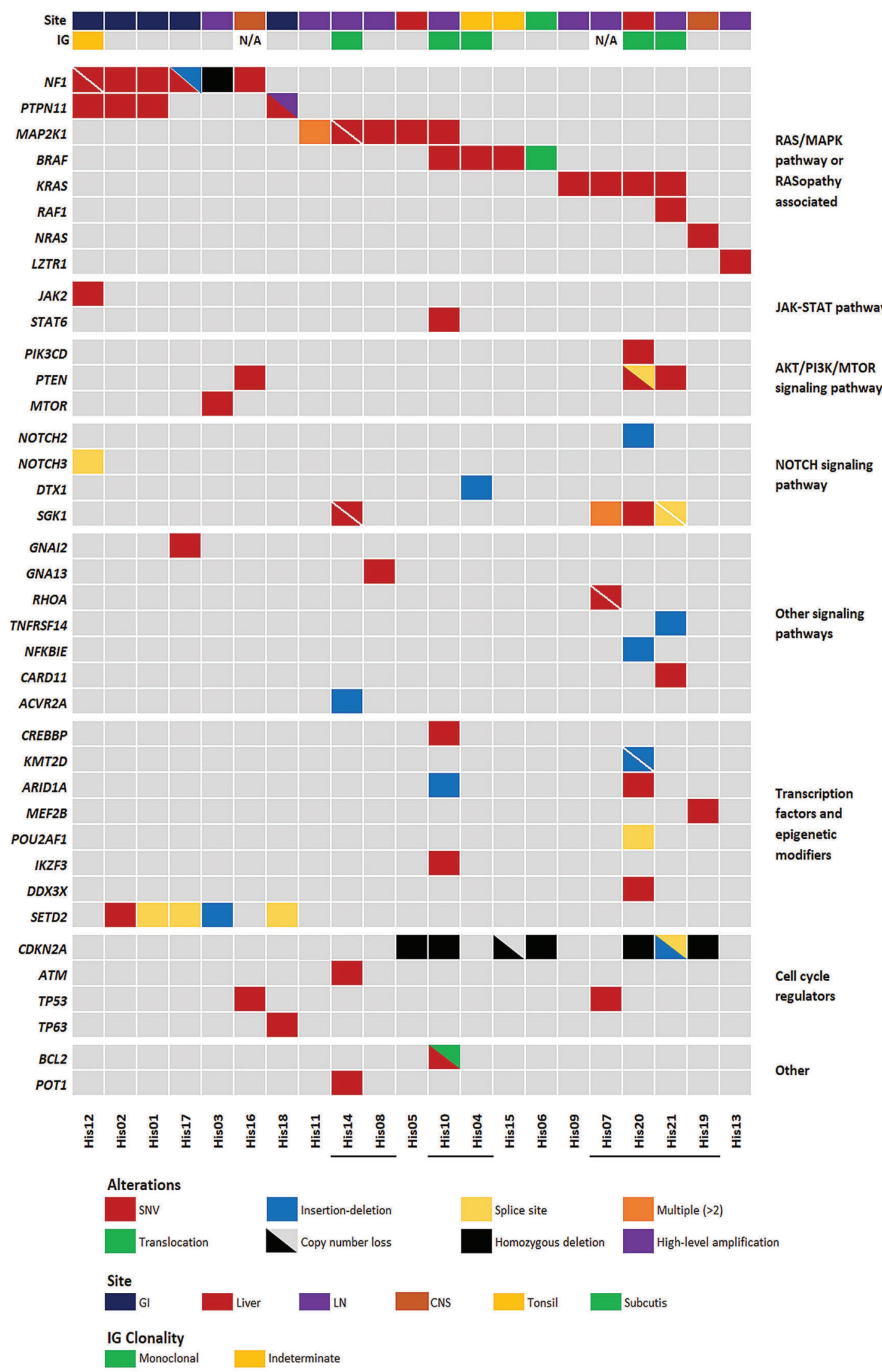

Figure 2. Selected molecular alterations in primary histiocytic sarcoma (pHS) cases. Genes are listed in the rows and organized by pathway or function. Samples are listed in columns. Site and IG gene rearrangement status are indicated in the top annotation bar. Underlined sample labels indicate a case with a B-cell lymphoma associated mutation or clonal IG gene rearrangement. Annotated focal copy number alterations and translocations were detected by OncoScan and/or verified by fluorescence in situ hybridization or polymerase chain reaction. Gl: gastrointestinal tract; LN: lymph node; CNS: central nervous system. 
A

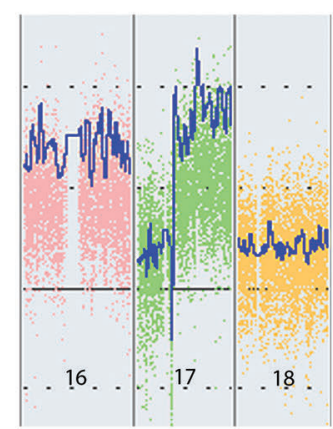

D

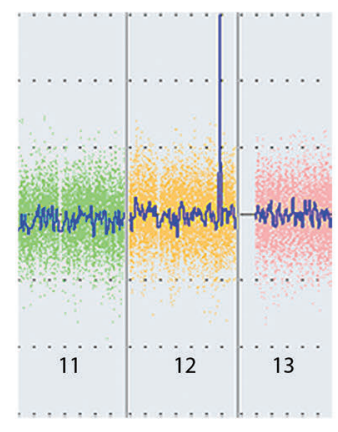

B

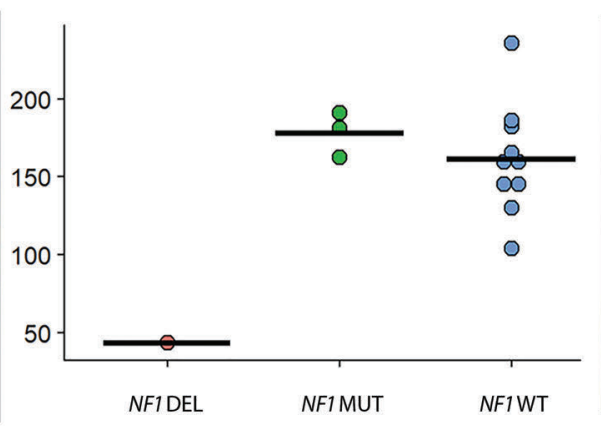

E

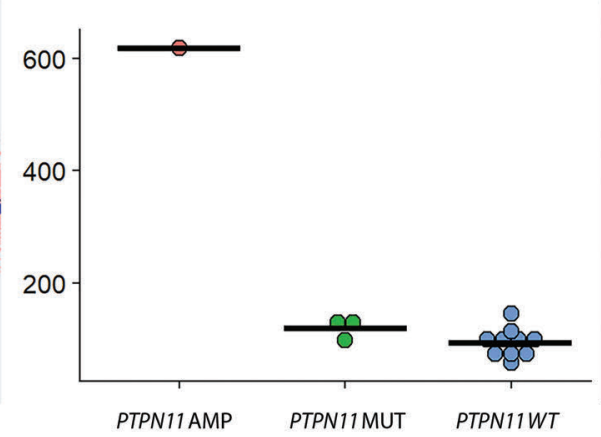

C

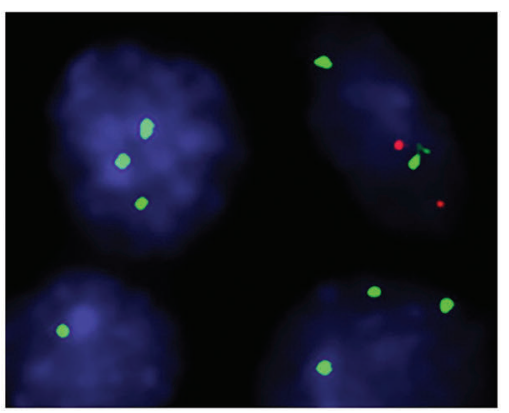

F

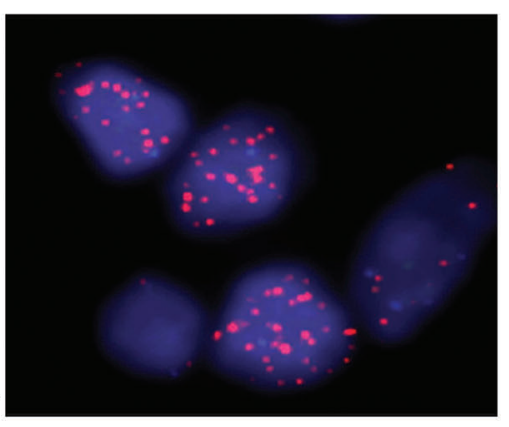

Figure 3. NF1 homozygous deletion. (A) Inverted peak in chromosome 17 consistent with homozygous deletion of NF1 (ChAS 3.3). (B) Reduction in NF1 transcript in the case with homozygous deletion (DEL) compared with NF1 mutated (MUT) and wild-type (WT) cases [normalized RNA-sequencing (RNA-Seq) count data]. (C) Fluorescence in situ hybridization (FISH) showing multiple copies of the CEP 17 probe (green) with loss of the NF1 probe (orange) (D-F). PTPN11 amplification. (D) Peak in chromosome 12 consistent with high-level amplification of PTPN11 (ChAS 3.3). (E) Increased PTPN11 transcript in the amplified (AMP) case compared with PTPN11 mutated (MUT) and wild-type (WT) cases (normalized RNA-Seq count data). (F) FISH showing multiple copies of the PTPN11 gene (orange) in a double minute pattern.

NF1 or PTPN11 mutations. MAP2K1 mutations were present in five cases and involved known hotspot regions in exons 2 and 3 , the negative regulatory region (p.F53L [His10, His14]; p.K57E [His05]) and the catalytic core domain (p.I103N [His14]; p.C121S [His08, His11]). ${ }^{30}$ As well as the mutation at p.C121S, case [His11] had two additional MAP2K1 mutations at p.Y125C and p.R181K. The functional consequences of these mutations are not known; however, the p.Y125C substitution also involved the catalytic core domain in the same allele as the pathogenic p.C121S mutation. One case with a MAP2K1 mutation had a co-occurring non-canonical BRAF mutation (p.G469V [His10]) and two additional cases had BRAF p.V600E [His04 and His15] mutations. Pathogenic KRAS mutations were detected in four cases (p.G12D [His21]; p.G12C [His09]; p.Q61H [His07] and p.A146V [His20]), one of which, [His21] also had a mutation in RAF1 (p.D486G). A single case had an NRAS mutation at p.Q61R [His19]. Finally, a mutation in LZTR1 (p.R118H) was identified in case [His13]. LZTR1 encodes an adaptor for CUL3 ubiquitin ligase complexes ${ }^{31}$ and is implicated in Noonan syndrome, ${ }^{32,33}$ malignancy ${ }^{31}$ and schwannomatosis. ${ }^{34}$ Mutations in LZTR1 have recently been shown to dysregulate RAS ubiquitination leading to increased RAS activity. ${ }^{35}$ The p.R118H mutation affects a conserved residue in the Kelch domain and is reported once in the COSMIC database. Mutations in LZTR1 are not typically associated with histiocytic tumors. This case met our inclusion criteria, but notably had some atypical features including aberrant expression of perforin and CD7 and a clonal TRG gene rearrangement.

Mutations involving the PI3K pathway were identified in four cases, including two with KRAS mutations and two with NF1 alterations, with mutations identified in PTEN (p.Q171* [His16]; p.D24H and c.79+1G>C [His20] and p.L140* [His21]), PIK3CD (p.E1021K [His20]) and MTOR (p.I2501F [His03]). Additional mutations in genes previously reported to be mutated in B-cell lymphomas were detected in SGK1 (p.R285K, p.I238T, p.H237Y, p.K213R and p.P147S [His07]; p.E162G and p.K136R [His14]; p.O125H [His20]; c.437+1G>A and c.362-1G>A [His21]), NOTCH2 (p.I2304fs [His20]), DTX1 (p.W37* [His04]), TNFRSF14 (p.T169fs [His21]), CARD11 (p.R1790 [His21]), NFKBIE (p.L410fs [His20]), GNA13 (p.F4V [His08]), POT1 (p.R273W [His14]) and BCL2 (p.E136D [His10]). ${ }^{36-40}$

In addition to mutations in the signaling pathways described above, mutations in epigenetic modifiers and/or transcription factors were detected in eight cases, including five with SETD2 mutations (c.7432-2A >C [His01]; p.V1820E [His02]; p.P132fs [His03]; c.4715+1G>T [His17] and c.7432-1G>A [His18]), two with ARID1A mutations (p.L2011fs [His10] and p.G2087R [His20]) and single cases with CREBBP (p.Y1433C [His10]), KMT2D (p.E2225fs and p.K1752fs [His20]), DDX3X (p.V206M [His20]), POU2AF1 (c.16+2T >G [His20]), IKZF3 (p.L162R [His10]), STAT6 
A

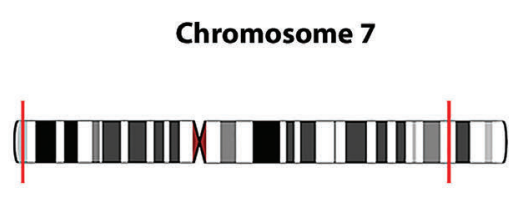

TTYH3

BRAF

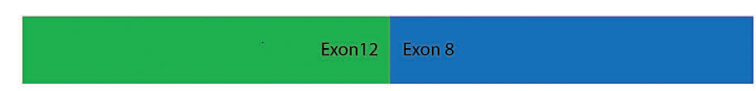

TTYH3-BRAF Fusion

C

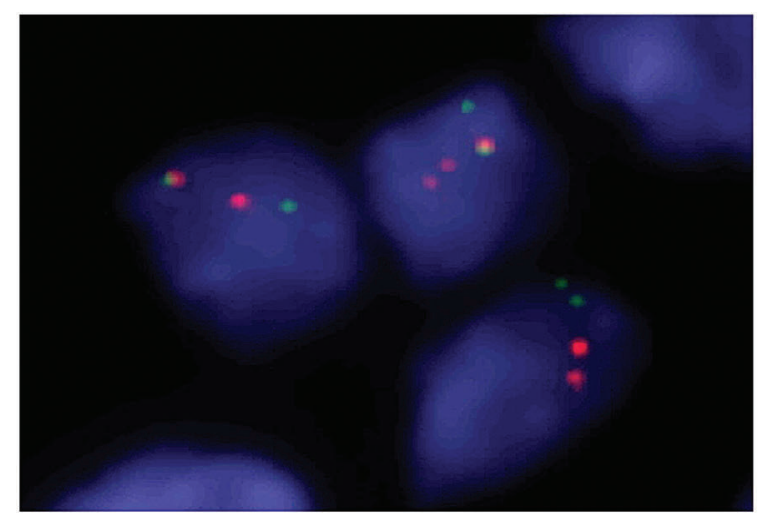

\section{B}

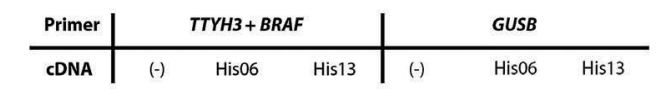

200

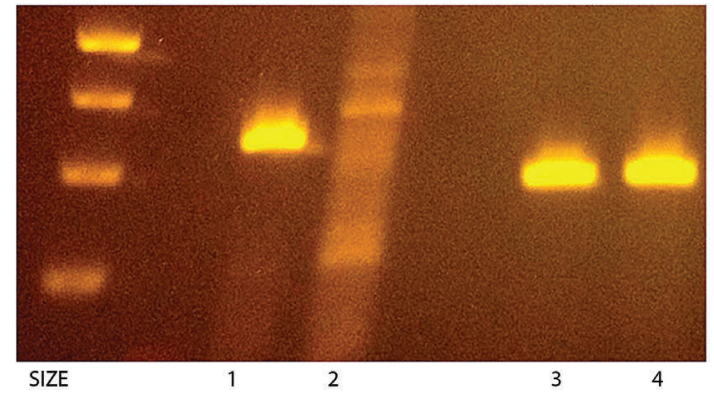

D

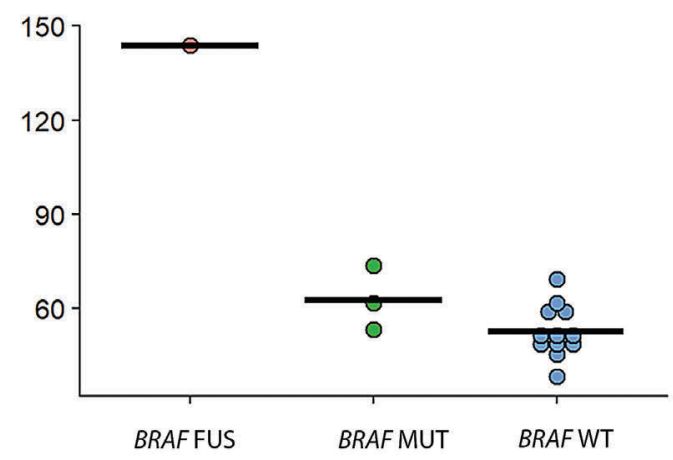

Figure 4. TTYH3-BRAF fusion. (A) Diagram of the intrachromosomal fusion on chromosome 7 of TTYH3 (exon 12) to BRAF (exon 8). (B) Gel electrophoresis of the reverse transcription polymerase chain reaction confirming the presence of the TTYH3-BRAF fusion in Lane 1 . (Lane 2 - Negative control; Lanes 3 and 4 - Positive controls). (C) Demonstration of split signals by fluorescence in situ hybridization, indicating a BRAF translocation (BRAF break apart probe, orange - 3'; green - 5'). (D) Increased BRAF transcript in the case with the fusion (FUS) compared with BRAF mutated (MUT) and wild-type (WT) cases (normalized RNA-Seq count data).

(p.D419G [His10]) and MEF2B (p.R64H [His19]) mutations. SETD2 mutations were exclusive to the NF1/PTPN11 mutated group, including one case found to have homozygous deletion of NF1 and one case with PTPN11 gene amplification (both described below), while the ARID1A, CREBBP, KMT2D, DDX3X, IKZF3, STAT6 and $M E F 2 B$ mutations were present in the NF1/PTPN11 wild-type group. Known pathogenic mutations in TP53 were identified in 2 of 21 cases (p.G245S [His07]; p.R175H [His16]).

\section{Copy number analysis shows additional alterations in NF1, PTPN11 and CDKN2A}

A homozygous deletion in the NF1 gene was identified in an additional case [His03] from a lymph node and confirmed using a fluorescence in situ hybridization (FISH) probe targeting the deleted area. RNA-Seq data showed markedly lower counts of NF1 transcript in this case in comparison to the other samples, consistent with loss of NF1 (Figure 3A-C). The three cases with a single NF1 mutation [His01, His02 and His16] showed loss-of-heterozygosity $(\mathrm{LOH})$ or copy number loss involving chromosome 17 including the NF1 gene.

Interestingly, a focal high-level amplification in chromosome 12 targeting PTPN11 was discovered in a further case [His18] involving the GI tract. This case harbored a known variant in the N-SH2 domain (p.E76K) of the amplified PTPN11 allele. In contrast to the other PTPN11 mutated cases, no mutation of NF1 was detected by exome sequencing. This high-level amplification was confirmed by FISH which showed multiple copies of the PTPN11 gene in the tumor cells in a double minute pattern. The amplified segment involved the entire PTPN11 gene, with the breakpoints identified by OncoScan in an adjacent gene, RPH3A, and 5' to PTPN11 involving HECTD 4 on the complementary strand. This event was associated with a dramatic increase in PTPN11 transcripts in comparison to the other cases (Figure 3D-F).

Cases with NF1/PTPN11 alterations had associated losses or LOH of chromosome 10 or $10 \mathrm{q}$ and chromosome 17 or $17 \mathrm{p}$ in 3 of 5 cases assessed by OncoScan [His01, His02, His12] and confirmed by FISH in two cases (Online Supplementary Figure S3A-C). Focal CDKN2A losses were present by OncoScan or confirmed by FISH in six cases that were NF1/PTPN11 wild-type [His05, His06, His10, His15, His19, His20] and included five cases with homozygous deletion [His05, His06, His10, His19, His20]. All homozygous deletions were confirmed by FISH (Online Supplementary Figure S3D and E). Both TP53 mutated cases had $\mathrm{LOH}$ involving the gene, with one NF1 mutated case [His16, not assessed by OncoScan] showing a near-haploid genome with loss of chromosome 17 and the second case [His07] showing $\mathrm{LOH}$ at chromosome $17 \mathrm{p}$.

\section{Identification of a novel TTYH3-BRAF fusion}

Fusion calling of RNA-Seq data identified a novel intrachromosomal fusion transcript between exon 12 of 


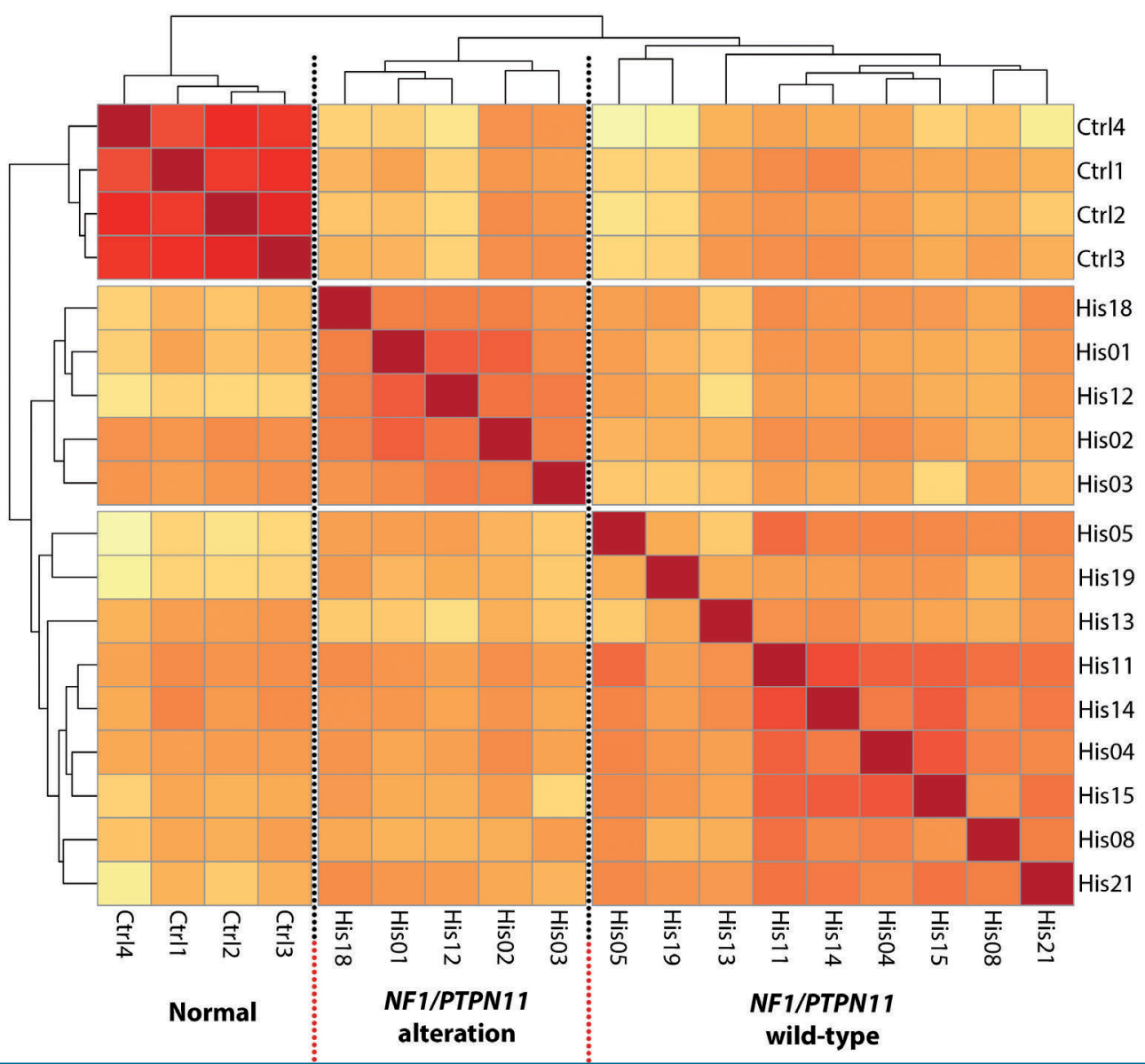

Figure 5. Exploratory analysis of RNA-sequencing (RNA-Seq) data. Unsupervised clustering based on transcriptome-wide gene expression and using Euclidean distance with complete linkage identifies three clusters corresponding to the normal samples, tumor samples with alterations in NF1/PTPN11 and the remaining NF1/PTPN11 wild-type tumor samples.

TTYH3 and exon 8 of BRAF on chromosome 7 in a case without any other known driver mutation [His06]. This novel TTYH3-BRAF fusion has a similar structure to other reported $B R A F$ fusions, ${ }^{41,42}$ with replacement of the RASbinding domain of BRAF by a 5' fusion partner, in this case, a chloride ion channel gene, TTYH3 (Figure 4A). The presence of the fusion transcript was confirmed by reverse transcription polymerase chain reaction (RT-PCR) using primers for exon 12 of TTYH3 and exon 8 of BRAF (Figure $4 \mathrm{~B}$ ), as well as by FISH (Figure 4C). Interestingly, RNA-Seq data showed markedly higher levels of $B R A F$ transcript as compared to all other samples, suggesting that the TTYH3 gene partner contributed an active promoter to the fusion gene (Figure 4D). TTYH3 was found to be highly expressed in all cases with RNA-Seq data (data not shown).

\section{Identification of two primary histiocytic sarcoma subgroups by whole transcriptome sequencing: association with NF1/PTPN11 mutational status}

We examined the gene expression profile of $\mathrm{pHS}$ through whole transcriptome sequencing of 17 of the tumor samples using four cases of reactive nodal histiocytic infiltrates as controls. Three of the 17 tumor samples initially sequenced were excluded from the differential expression analysis as they failed quality control metrics and/or were outliers within the tumor group [His10] or within the data as a whole [His06, His09]
(Online Supplementary Figure S4). Re-clustering segregated the remaining samples into three groups: normal controls (4 samples), cases with NF1 or PTPN11 abnormalities (5 samples), and a third heterogeneous group comprising NF1/PTPN11 wild-type cases (9 samples) (Figure 5).

\section{Gene set enrichment analysis shows enrichment of cell cycle processes in cases without NF1/PTPN11 abnormalities}

To better understand the potential biological significance of the two pHS subgroups, we performed gene set enrichment analysis using EGSEA. This analysis showed significant enrichment of cell cycle pathway and cell proliferation gene sets in the NF1/PTPN11 wild-type tumor samples relative to samples with $N F 1 / P T P N 11$ alterations. Ki67 immunohistochemistry was performed on a subset of cases and confirmed the lower proliferation rate in the NF1/PTPN11 subgroup (Figure 6 and Online Supplementary Table S3).

The two tumor subgroups were also evident in the differential expression analysis comparing the normal and tumor samples when the genes with significant differential expression (FDR $<0.05$, absolute log fold change $>1$ ) were visualized across samples (Online Supplementary Table S5 and Online Supplementary Figure S5). We took advantage of this to explore the possibility that disease site might be influencing the clustering of the tumor sub- 
A

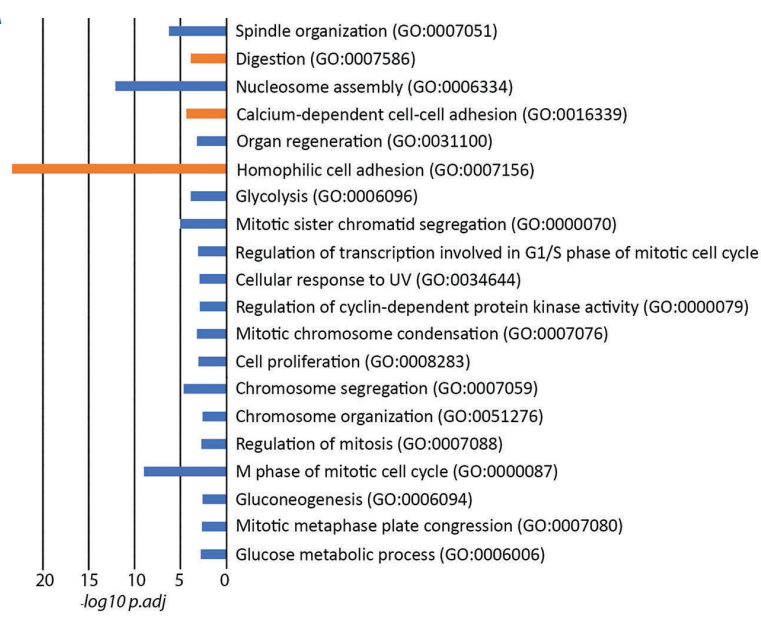

C

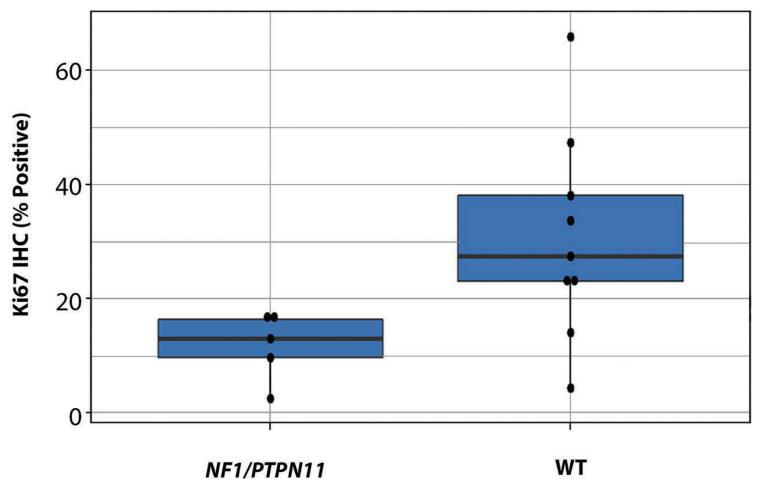

B

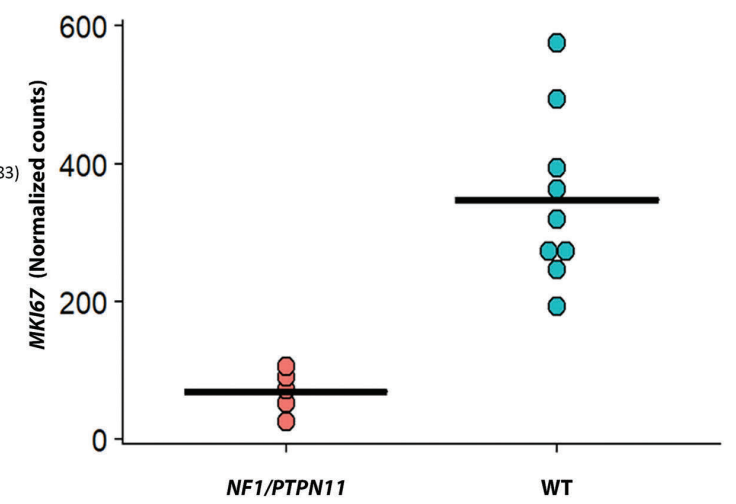

D

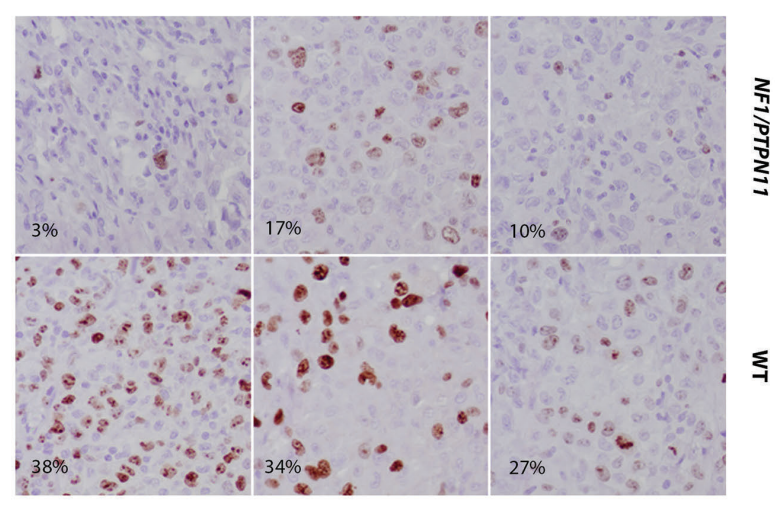

Figure 6. Cell cycle and proliferation. (A) The -log10 adjusted P-values of the top 20 GO Biological Processes (GeneSetDB Gene Ontology) by gene set enrichment analysis using EGSEA, sorted by average rank. There is enrichment for cell cycle and proliferation-related processes in the NF1/PTPN11 wild-type (WT) cases (blue) relative to the cases with NF1/PTPN11 alterations. (B) Normalized counts from RNA-sequencing data showing differences in MKI67 transcript between the NF1/PTPN11 altered and NF1/PTPN11 WT groups. (C) Bar plot of the difference in proliferation index between the tumor subgroups by Ki67 immunohistochemistry. (D) Photomicrograph showing immunohistochemical expression of Ki67 in the tumor subgroups.

groups, as four of the five NF1/PTPN11 samples subjected to unsupervised clustering were GI excisions. When we excluded genes associated with GI site from this tumor versus normal comparison, the two tumor subclusters were unaffected; however, a set of genes that were more clearly differentially expressed between the tumor subgroups were revealed that upon removal resulted in the elimination of the tumor subgroups. Functional enrichment of this set of genes using ToppFun (https://toppgene.cchmc.org) showed enrichment for cell cycle processes, supporting the EGSEA result and the interpretation that the observed clustering was related to the difference in cell cycle processes between tumor groups rather than the tumor site (Online Supplementary Tables S6-S8 and Online Supplementary Figures S6 and S7).

\section{Correlation of clonal IG rearrangement status and B-cell associated mutations with NF1/PTPN11 wild-type status}

Clonal analysis to detect rearrangements of IG and TRG genes was performed on 19 of 21 cases, including 6 of 7 NF1/PTPN11 cases and 13 of 14 NF1/PTPN11 wildtype cases. Five cases had clonal rearrangement of the IGH and/or the IGK locus [His04, His10, His14, His20, His21], while two cases (including one with IG rearrangement) showed rearrangements of the TRG locus [His13, His20]. One case was indeterminate for a significant clonal IG rearrangement [His12]. All five clonally rearranged cases for IG were NF1/PTPN11 wild-type and all had additional mutations in transcriptional regulators and/or signaling pathway genes previously reported altered in B-cell lymphoma (see above). Three additional cases in the NF1/PTPN11 wild-type group had at least one B-cell associated gene mutation [His07, His08 and His19]. IGH/BCL2 (MBR) translocation analysis was positive in 1 of 17 cases [His10] (Online Supplementary Table S3). In total, 8 of the 14 cases in the NF1/PTPN11 wild-type subgroup had clonal IG gene rearrangements or mutations in genes reported to be mutated in B-cell lymphomas (Figure 2).

\section{Discussion}

Our study furthers the current understanding of the genomic landscape of primary HS through integration of whole exome sequencing and gene expression analysis. It confirms the central role of the RAS/MAPK pathway in the pathogenesis of $\mathrm{pHS}$, with RAS pathway abnormalities identified in all cases in this study. Moreover, it identifies 
two molecular subgroups based on the presence or absence of NF1/PTPN11 alterations and prevalence of SETD2 mutations, and independently through unsupervised clustering of RNA-Seq data. In addition, our study identifies novel mechanisms of RAS/MAPK pathway activation, including a previously unreported intrachromosomal fusion between TTYH 3 and BRAF that preserves the BRAF kinase domain, and high-level PTPN11 amplification. Perhaps the most surprising finding of our study was the discovery of the NF1/PTPN11 subgroup with its distinct molecular characteristics and tissue site of involvement. In contrast to the NF1/PTPN11 wild-type subgroup, none of these cases harbored abnormalities in genes associated with B-cell lymphomas beyond SETD2 or had clonal IG rearrangements. GSEA revealed that this subgroup was characterized by a relative loss of gene sets related to cellular proliferation and the cell cycle compared to those harboring other RAS/MAPK alterations, a finding supported by Ki67 immunohistochemistry.

The majority of the NF1/PTPN11 mutant cases had more than one MAPK pathway activating mutation. Three of the seven cases had co-occurring NF1 and PTPN11 mutations, while a fourth case had a co-occurring mutation in GNAI2 involving a codon previously shown to activate the MAPK pathway. ${ }^{29}$ Additionally, while the remaining PTPN11 mutated case did not have a co-occurring $R A S$ mutation, it did have high-level amplification of the mutated PTPN11 allele. In NF1 mutant melanoma, the frequent presence of a second gene mutation often involving PTPN11 (or another RASopathy gene) has led to the suggestion that NF1 inactivation is insufficient to cause full activation of the downstream MAPK pathway and tumorigenesis. ${ }^{43}$ This hypothesis has been given further credence by recent data showing that NF1 loss-of-function mutant cell lines are dependent on SHP2 (encoded by PTPN11) mediated signaling for oncogenic RAS/MAPK pathway activation, ${ }^{44}$ raising the possibility that activating mutations of PTPN11 may synergize with NF1 loss-of-function mutations to further potentiate the oncogenic activity of the pathway.

In contrast to the NF1/PTPN11 positive subgroup, the NF1/PTPN11 wild-type cluster was comprised primarily of cases with prototypic RAS/MAPK pathway activating mutations involving KRAS, NRAS, BRAF and MAP2K1. Interestingly, eight of the 14 cases in this subgroup contained IG gene rearrangements and/or additional mutations in genes commonly associated with B-cell lymphoproliferative disorders. These included one or more mutations in epigenetic regulators, transcription factors or signaling pathway genes, including CREBBP, KMT2D, DDX3X, ARID1A, MEF2B, SGK1, TNFRSF14, DTX1, GNA13, STAT6 and $C A R D 11{ }^{36-40}$ Clonal IG rearrangements were identified in five cases and a BCL2 gene rearrangement was identified in one case, while neither were definitively detected in the NF1/PTPN11 subgroup. In our series, it is worth noting that none of our cases had evidence of a concurrent or previous lymphoma, although we cannot exclude the possibility of an occult or unreported B-cell lymphoma being present. The finding of additional mutations associated with B-cell lymphomas and clonal IG gene rearrangements suggests that some cases of NF1/PTPN11 wild-type pHS may be similar in origin to the sHS that are associated with B-cell malignancies, which often share IG gene rearrangements with the associated B-cell lymphoma. ${ }_{5,6}$ This overlap has also been recently reported by Shanmugam et al. ${ }^{18}$ In their series, they showed enrichment for a mutational signature resembling aberrant somatic hypermutation in cases that had a history of B-cell lymphoma or that had mutations in genes that are frequently mutated in B-cell lymphoma. Interestingly, they also found recurrent CDKN2A alterations that were more frequent in cases with a history of $B$ cell lymphoma or the aberrant somatic hypermutation signature. Similarly, our study identified a high frequency of focal CDKN2A losses/alterations in the NF1/PTPN11 wildtype subgroup which, in our cohort, frequently had molecular alterations associated with B-cell lymphoma.

Concurrent mutations in RAS/MAPK pathway genes were less common in the NF1/PTPN11 wild-type group, occurring in 4 of 14 cases with alterations. Two cases had concurrent mutations in MAP2K1 [His11 and His14]. The other two had co-occurring BRAF (p.G469V) and MAP2K1 (p.F53L) mutations [His10] or KRAS (p.G12D) and RAF1 (p.D486G) mutations [His21]. These data are consistent with the limited published data in HS in which reported occurrences of multiple RAS/MAPK pathway mutations tend to manifest as co-occurring MAP2K1 mutations ${ }^{18}$ or involve atypical $B R A F$ mutations, with co-occurring $B R A F$ (p.G464V) and KRAS (p.Q61H), ${ }^{45}$ BRAF (p.D594N) and KRAS (p.A146T), ${ }^{18} B R A F$ (p.G469R) and NF1 (p.W2229*) and $B R A F$ (p.F595L) and HRAS (p.Q61R) mutations ${ }^{46}$ described. Interestingly, in the latter case the unusual $B R A F$ mutation was shown to have weak oncogenic activity requiring the co-operation of the HRAS mutation for full activity.

Two of the analytical challenges in our study included the lack of available matched germline samples in all but three cases, and the possibility that over-representation of GI site in the NF1/PTPN11 group could bias the RNA-Seq clustering. To exclude as many germline SNPs as possible we filtered all variants using stringent criteria for their representation in control populations (gnomAD) ${ }^{47}$ and took CADD scores, ${ }^{20}$ as well as presence in the Catalogue of Somatic Mutations in Cancer (COSMIC) ${ }^{48}$ into consideration. In assessing potential site bias in gene expression clustering, we found that the separation of the tumor samples into the subgroups in the differential expression analysis was influenced by the removal of cell cycle-related genes but not by exclusion of GI site-associated genes. This, in addition to the similar mutational alterations in the cases, suggests that the clustering observed occurs independently of site.

In conclusion, our study provides further insight into the molecular pathogenesis of pHS. We show frequent mutations and alterations in genes of the RAS/MAPK pathway, suggesting that patients could potentially benefit from genomic evaluation and targeted therapy, and we report a distinct molecular subtype of pHS that correlates with the NF1/PTPN11 status of the tumor and frequently involves the GI tract. Finally, we also identify a subset of NF1/PTPN11 wild-type cases with mutations in B-cell lymphoma associated genes and/or clonal IG gene rearrangements. The identification of molecular subtypes of primary histiocytic sarcoma may prove to have clinical relevance in future studies.

\section{Acknowledgments}

The authors would like to thank Andrea O'Hara (BioDiscovery) and Karen Gustashaw (Thermo Fisher Scientific) for their assistance and software support in the interpretation of the OncoScan data; Arati Raziuddin, Xiaolin Wu, Jyoti Shetty, Bao Tran and Nina Bubunenko (Frederick National Laboratory for Cancer Research, Leidos Biomedical Research, Inc.) for per- 
forming the library preparation, exome and RNA-Seq and OncoScan assay; Louis M. Staudt (Lymphoid Malignancies Branch, Center for Cancer Research, National Cancer Institute) for his critical reading of our manuscript.

\section{Funding}

This work was supported by the intramural research program of the Center for Cancer Research, National Cancer Institute, National Institutes of Health.

\section{References}

1. Swerdlow SH, Campo E, Harris NL, et al., eds. WHO Classification of Tumours of Haematopoietic and Lymphoid Tissues. Revised 4th Edition ed. Lyon, France: IARC, 2017.

2. Kommalapati A, Tella SH, Durkin M, Go RS, Goyal G. Histiocytic sarcoma: a populationbased analysis of incidence, demographic disparities, and long-term outcomes. Blood. 2018;131(2):265-268.

3. Facchetti F, Pileri SA, Lorenzi L, et al. Histiocytic and dendritic cell neoplasms: what have we learnt by studying 67 cases. Virchows Arch. 2017;471(4):467-489.

4. Emile JF, Abla O, Fraitag S, et al. Revised classification of histiocytoses and neoplasms of the macrophage-dendritic cell lineages. Blood. 2016;127(22):2672-2681.

5. Feldman AL, Arber DA, Pittaluga S, et al. Clonally related follicular lymphomas and histiocytic/dendritic cell sarcomas: evidence for transdifferentiation of the follicular lymphoma clone. Blood. 2008;111(12):54335439.

6. Shao H, Xi L, Raffeld M, et al. Clonally related histiocytic/dendritic cell sarcoma and chronic lymphocytic leukemia/small lymphocytic lymphoma: a study of seven cases. Mod Pathol. 2011;24(11):1421-1432.

7. Feldman AL, Minniti C, Santi M, Downing JR, Raffeld M, Jaffe ES. Histiocytic sarcoma after acute lymphoblastic leukaemia: a common clonal origin. Lancet Oncol. 2004:5(4):248-250.

8. Soslow RA, Davis RE, Warnke RA, Cleary ML, Kamel OW. True histiocytic lymphoma following therapy for lymphoblastic neoplasms. Blood. 1996;87(12):5207-5212.

9. Alten J, Klapper W, Leuschner I, et al. Secondary histiocytic sarcoma may cause apparent persistence or recurrence of minimal residual disease in childhood acute lymphoblastic leukemia. Pediatr Blood Cancer. 2015;62(9):1656-1660.

10. Hure MC, Elco CP, Ward D, et al. Histiocytic sarcoma arising from clonally related mantle cell lymphoma. J Clin Oncol. 2012;30(5):e4953.

11. Brunner P, Rufle A, Dirnhofer S, et al. Follicular lymphoma transformation into histiocytic sarcoma: indications for a common neoplastic progenitor. Leukemia. 2014;28(9):1937-1940.

12. Chen W, Lau SK, Fong D, et al. High frequency of clonal immunoglobulin receptor gene rearrangements in sporadic histiocytic/dendritic cell sarcomas. Am J Surg Pathol. 2009:33(6):863-873.

13. Hayase E, Kurosawa M, Yonezumi M, Suzuki S, Suzuki H. Aggressive sporadic histiocytic sarcoma with immunoglobulin heavy chain gene rearrangement and $\mathrm{t}(14$;8). Int J Hematol. 2010;92(4):659-663.

14. Emile JF, Diamond EL, Helias-Rodzewicz Z, et al. Recurrent RAS and PIK3CA mutations in Erdheim-Chester disease. Blood. 2014;124(19):3016-3019.

15. Chakraborty R, Hampton OA, Shen X, et al. Mutually exclusive recurrent somatic mutations in MAP2K1 and BRAF support a central role for ERK activation in $\mathrm{LCH}$ pathogenesis.
Blood. 2014;124(19):3007-3015.

16. Diamond EL, Durham BH, Haroche J, et al. Diverse and targetable kinase alterations drive histiocytic neoplasms. Cancer Discov. 2016;6(2):154-165.

17. Badalian-Very G, Vergilio JA, Degar BA, et al. Recurrent BRAF mutations in Langerhans cell histiocytosis. Blood. 2010; 116(11):19191923.

18. Shanmugam V, Griffin GK, Jacobsen ED, Fletcher CDM, Sholl LM, Hornick JL. Identification of diverse activating mutations of the RAS-MAPK pathway in histiocytic sarcoma. Mod Pathol. 2019:32(6):830-843.

19. Sondka Z, Bamford S, Cole CG, Ward SA, Dunham I, Forbes SA. The COSMIC Cancer Gene Census: describing genetic dysfunction across all human cancers. Nat Rev Cancer. 2018;18(11):696-705.

20. Rentzsch P, Witten D, Cooper GM, Shendure J, Kircher M. CADD: predicting the deleteriousness of variants throughout the human genome. Nucleic Acids Res. 2019;47(D1):D886-D894.

21. Robinson JT, Thorvaldsdottir $\mathrm{H}$, Winckler W, et al. Integrative genomics viewer. Nat Biotechnol. 2011;29(1):24-26.

22. Alhamdoosh M, Ng M, Wilson NJ, et al. Combining multiple tools outperforms individual methods in gene set enrichment analyses. Bioinformatics. 2017;33(3):414424.

23. Talevich E, Shain AH, Botton T, Bastian BC. CNVkit: genome-wide copy number detection and visualization from targeted DNA sequencing. PLoS Comput Biol. 2016; 12(4):e1004873

24. Riester M, Singh AP, Brannon AR, et al. PureCN: copy number calling and SNV classification using targeted short read sequencing. Source Code Biol Med. 2016;11:13.

25. Kumar P, Henikoff S, Ng PC. Predicting the effects of coding non-synonymous variants on protein function using the SIFT algorithm. Nat Protoc. 2009;4(7):1073-1081.

26. Adzhubei IA, Schmidt S, Peshkin L, et al. A method and server for predicting damaging missense mutations. Nat Methods. 2010;7(4):248-249.

27. Kratz CP, Niemeyer CM, Castleberry RP, et al. The mutational spectrum of PTPN11 in juvenile myelomonocytic leukemia and Noonan syndrome/myeloproliferative disease. Blood. 2005;106(6):2183-2185.

28. Tajan M, de Rocca Serra A, Valet P, Edouard T, Yart A. SHP2 sails from physiology to pathology. Eur J Med Genet. 2015; 58(10):509-525.

29. Nairismagi ML, Tan J, Lim JQ, et al. JAKSTAT and G-protein-coupled receptor signaling pathways are frequently altered in epitheliotropic intestinal T-cell lymphoma. Leukemia. 2016;30(6):1311-1319.

30. Brown NA, Furtado LV, Betz BL, et al. High prevalence of somatic MAP2K1 mutations in BRAF V600E-negative Langerhans cell histiocytosis. Blood. 2014;124(10):1655-1658.

31. Frattini V, Trifonov V, Chan JM, et al. The integrated landscape of driver genomic alterations in glioblastoma. Nat Genet. 2013:45(10):1141-1149.

32. Johnston JJ, van der Smagt JJ, Rosenfeld JA, et al. Autosomal recessive Noonan syndrome associated with biallelic LZTR1 variants. Genet Med. 2018;20(10):1175-1185.

33. Tidyman WE, Rauen KA. Expansion of the RASopathies. Curr Genet Med Rep. 2016; 4(3):57-64.

34. Piotrowski A, Xie J, Liu YF, et al. Germline loss-of-function mutations in LZTR1 predispose to an inherited disorder of multiple schwannomas. Nat Genet. 2014;46(2):182187.

35. Steklov M, Pandolfi S, Baietti MF, et al Mutations in LZTR1 drive human disease by dysregulating RAS ubiquitination. Science. 2018:362(6419):1177-1182.

36. Karube K, Enjuanes A, Dlouhy I, et al. Integrating genomic alterations in diffuse large B-cell lymphoma identifies new relevant pathways and potential therapeutic targets. Leukemia. 2018;32(3):675-684.

37. Chapuy B, Stewart C, Dunford AJ, et al Molecular subtypes of diffuse large B cell lymphoma are associated with distinct pathogenic mechanisms and outcomes. Nat Med. 2018;24(5):679-690.

38. Reddy A, Zhang J, Davis NS, et al. Genetic and Functional Drivers of Diffuse Large B Cell Lymphoma. Cell. 2017;171(2):481494.e15.

39. Schmitz R, Wright GW, Huang DW, et al Genetics and pathogenesis of diffuse large $\mathrm{B}$ cell lymphoma. N Engl J Med. 2018; 378(15):1396-1407.

40. Landau DA, Sun C, Rosebrock D, et al. The evolutionary landscape of chronic lymphocytic leukemia treated with ibrutinib targeted therapy. Nat Commun. 2017; 8(1):2185.

41. Ross JS, Wang K, Chmielecki J, et al. The distribution of BRAF gene fusions in solid tumors and response to targeted therapy. Int J Cancer. 2016;138(4):881-890

42. Ciampi R, Knauf JA, Kerler R, et al. Oncogenic AKAP9-BRAF fusion is a novel mechanism of MAPK pathway activation in thyroid cancer. J Clin Invest. 2005; 115(1):94101.

43. Krauthammer M, Kong Y, Bacchiocchi A, et al. Exome sequencing identifies recurrent mutations in NF1 and RASopathy genes in sun-exposed melanomas. Nat Genet. 2015 47(9):996-1002

44. Nichols RJ, Haderk F, Stahlhut C, et al. RAS nucleotide cycling underlies the SHP2 phosphatase dependence of mutant BRAF-, NF1and RAS-driven cancers. Nat Cell Biol. 2018;20(9):1064-1073.

45. Liu $\mathrm{Q}$, Tomaszewicz $\mathrm{K}$, Hutchinson $\mathrm{I}$ Hornick JL, Woda B, Yu H. Somatic mutations in histiocytic sarcoma identified by next generation sequencing. Virchows Arch. 2016;469(2):233-241.

46. Kordes $M$, Roring $M$, Heining $C$, et al. Cooperation of BRAF(F595L) and mutant HRAS in histiocytic sarcoma provides new insights into oncogenic BRAF signaling. Leukemia. 2016;30(4):937-946.

47. Lek M, Karczewski KJ, Minikel EV, et al. Analysis of protein-coding genetic variation in 60,706 humans. Nature. 2016;536(7616):285-291.

48. Forbes SA, Beare D, Boutselakis $\mathrm{H}$, et al COSMIC: somatic cancer genetics at highresolution. Nucleic Acids Res. 2017; 45(D1):D777-D783 